\title{
How to make sticky surfaces slippery: Contact angle hysteresis in electrowetting with alternating voltage
}

\author{
F. Li and F. Mugele ${ }^{a)}$ \\ Physics of Complex Fluids, Faculty of Science and Technology, Institute of Mechanics, Process and Control \\ Twente, MESA + Institute for Nanotechnology, University of Twente, P.O. Box 217, 7500 AE Enschede, \\ The Netherlands
}

(Received 8 March 2008; accepted 28 May 2008; published online 20 June 2008)

Contact angle hysteresis caused by random pinning forces is a major obstacle in moving small quantities of liquid on solid surfaces. Here, we demonstrate that the contact angle hysteresis for sessile drops in electrowetting almost disappears with increasing alternating voltage, whereas for direct voltage it remains constant. This observation is explained in terms of a balance of surface tension, pinning, and (time-dependent) electrostatic forces at the contact line. (C) 2008 American Institute of Physics. [DOI: 10.1063/1.2945803]

Large water drops roll down windows on rainy days, where as small ones remain stuck. This behavior is due the increasing importance of random pinning forces caused by surface heterogeneities at smaller scales. ${ }^{1-3}$ In microfluidic applications, this gives rise to undesirable threshold forces for drop displacement. ${ }^{4-9}$ To achieve efficient motion, one has to either prepare samples with sufficiently low heterogeneity, i.e., with minimum contact angle (c.a.) hysteresis ${ }^{9}$ or to provide sufficient "vibrational energy" to overcome the pinning forces, as originally suggested by Johnson and Dettre. ${ }^{10}$ Using the latter concept, various groups succeeded in reducing the effective c.a. hysteresis (e.g., Refs. 11-13) and in inducing droplet motion ${ }^{14,15}$ by mechanical shaking. From the perspective of miniaturization, however, this approach seems dissatisfying in view of the decreasing inertia at small scales.

In this letter, we show that electric fields in an electrowetting (EW) configuration provide an exquisite way of overcoming pinning forces at the contact line (CL). In contrast to mechanical shaking, EW gives rise to well-controlled forces localized at the CL. ${ }^{16}$ Measuring advancing and receding c.a.'s as a function of both alternating current(ac) and direct current (DC) voltages, we establish a quantitative relation between these forces and the observed c.a. hysteresis.

c.a. measurements were performed using sessile drops of a $\mathrm{NaCl}$ solution (electrical conductivity: $1 \mathrm{mS} / \mathrm{cm}$ ) in deionized water in ambient air using a commercial c.a. goniometer [OCA30 by Dataphysics (Germany)] equipped with a motorized syringe pump. Side view images of the drops were analyzed to determine the c.a. $\theta$ of the drop with an accuracy of $\pm 1^{\circ}$. Reported c.a. values are averages of the independently fitted left and right edges of the drop. The drop volume $V[1-10 \mu \mathrm{l}]$ was varied at a constant rate $(0.1 \mu \mathrm{l} / \mathrm{s})$ sufficiently low to avoid dynamic c.a. effects. Drops were deposited onto glass substrates with conductive indium-tinoxide (ITO) layers covered with an insulating layer of Teflon AF 1600 (Dupont). The Teflon layers with a thickness $d$ of 3-5 $\mu \mathrm{m}$ were produced by dip-coating from a $6 \%$ solution in the standard solvent FC-75 and annealed in a vacuum oven at $300{ }^{\circ} \mathrm{C}$ for $30 \mathrm{~min}$. The drops were electrically

\footnotetext{
${ }^{\text {a) }}$ Author to whom correspondence should be addressed. Electronic mail:
} f.mugele@utwente.nl. grounded via the immersed syringe needle (diameter: $0.25 \mathrm{~mm}$ ). A variable voltage $U\left[0-160 \mathrm{~V}_{\mathrm{rms}}\right.$ (root mean square)] at frequencies $\nu=0-10 \mathrm{kHz}$ was applied to the ITO layer on the substrate. The experimental setup is sketched in Fig. 1(a).

Figure 2 shows experimental raw data of c.a. hysteresis curves. Upon inflating the drop volume (starting at the first arrow), the CL initially remained pinned and $\theta$ increased from its arbitrary initial value up to a maximum value, the advancing c.a. $\theta_{a}$, whereupon the $\mathrm{CL}$ advanced with $\theta=\theta_{a}$. Upon reversing the pumping direction (second arrow), $\theta$ initially decreased and the CL remained pinned until $\theta$ reached the receding c.a. $\theta_{r}$. This qualitative behavior was observed for both ac and dc voltages. The quantitative dependence of $\theta_{a}$ and $\theta_{r}$ on the voltage, however, was quite different: In the ac case [Fig. 2(a)], $\theta_{a}$ decreased upon increasing $U$, while the receding branch initially displayed a very weak voltage dependence. Only for the highest voltages, both $\theta_{a}$ and $\theta_{r}$ decreased at the same rate. As a consequence, the c.a. hysteresis $\Delta \theta=\theta_{a}-\theta_{r}$ decreased from approximately $13^{\circ}$ to $2^{\circ}$

a)
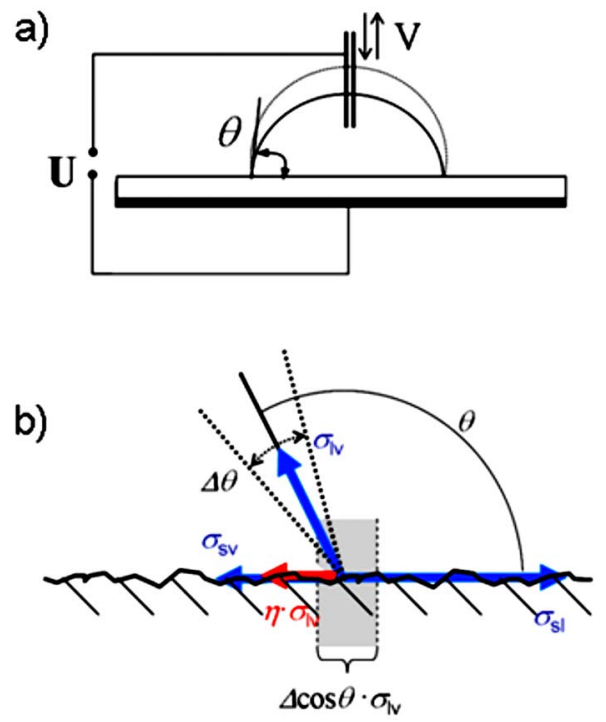

FIG. 1. (Color online) (a) Experimental setup. Thick solid line: bottom electrode covered by a shaded insulating layer (thickness not to scale). (b) Force balance at the contact line including electrostatic force and range of pinning forces (gray band). 

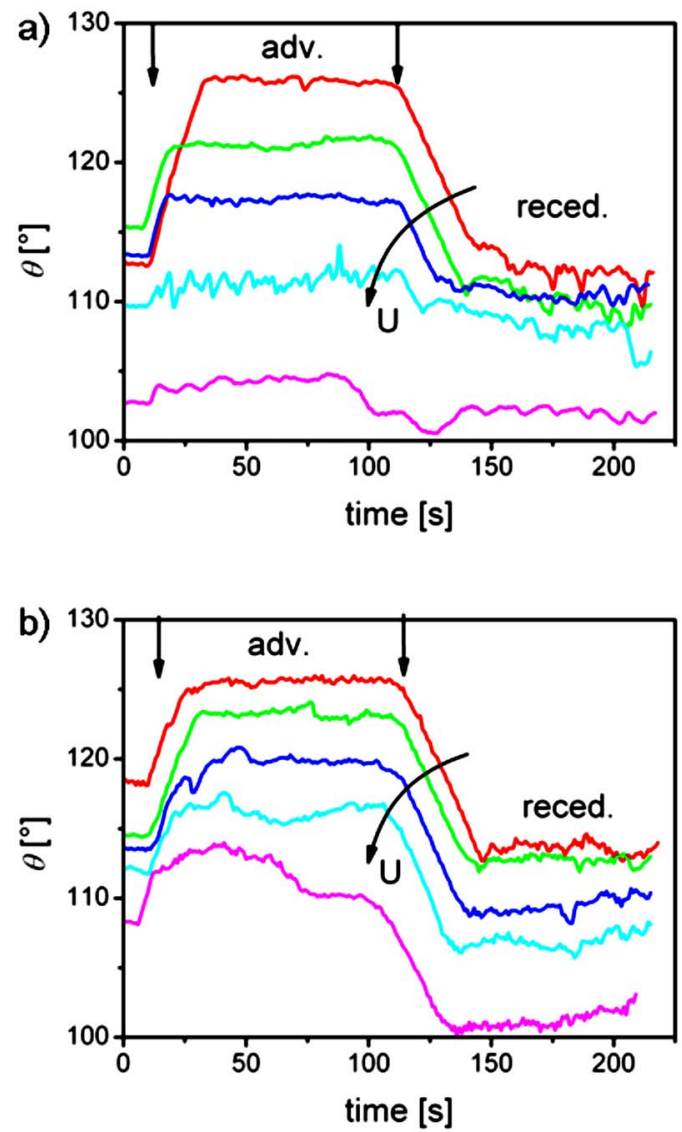

FIG. 2. (Color online) Advancing and receding contact lines for (a) ac voltage at $f=1 \mathrm{kHz}$ and (b) dc voltage. The applied voltage increases from top to bottom: $0,20,40,60$, and $80 \mathrm{~V}$.

from zero to the highest voltage. This behavior was observed for all ac frequencies between 0.2 and $10 \mathrm{kHz}$. In contrast, for dc voltage, both $\theta_{a}$ and $\theta_{r}$ decrease in the same way upon increasing $U$.

Overall, a decrease of both $\theta_{a}$ and $\theta_{r}$ is, in fact, expected since the equilibrium c.a. is known to decrease with increasing applied voltage following the EW equation

$$
\cos \theta=\cos \theta_{Y}+\frac{\varepsilon_{0} \varepsilon_{d}}{2 d \sigma_{\mathrm{lv}}} U^{2}=\cos \theta_{Y}+\eta,
$$

which is valid in the low voltage range investigated here (see Ref. 17 for a review); $\varepsilon_{0}$ is the vacuum permittivity, $\varepsilon_{d}=2$ is the dielectric constant of the insulating layer, and $\sigma_{\mathrm{lv}}$ $=72 \mathrm{~mJ} / \mathrm{m}^{2}$ is the surface tension. For ac voltage, the rms value of the applied voltage has to be inserted for $U$. In Eq. (1), we introduced the dimensionless EW number $\eta$ $=\varepsilon_{0} \varepsilon_{d} U^{2} /\left(2 d \sigma_{\mathrm{lv}}\right)$, which measures the strength of the electrostatic forces with respect to the surface tension.

In Fig. 3, we plot the cosines of both $\theta_{a}$ and $\theta_{r}$ as a function of the EW number, as suggested by Eq. (1). For dc voltage (open symbols), both $\theta_{a}$ and $\theta_{r}$ follow rather well the behavior predicted for the equilibrium c.a. [Eq. (1)] for all voltages. For ac voltage (full symbols), however, $\cos \theta_{a}$ (triangles) increases stronger than expected for low $\eta$, while $\cos \theta_{r}$ (squares) remains almost constant. Above a certain threshold value $\tilde{\eta}(\approx 0.1$ for the present data), both ac curves adopt the same slope as the dc data, which is equal to unity within the error of the insulator thickness calibration. Note that the c.a. hysteresis for ac voltage decreases substantially

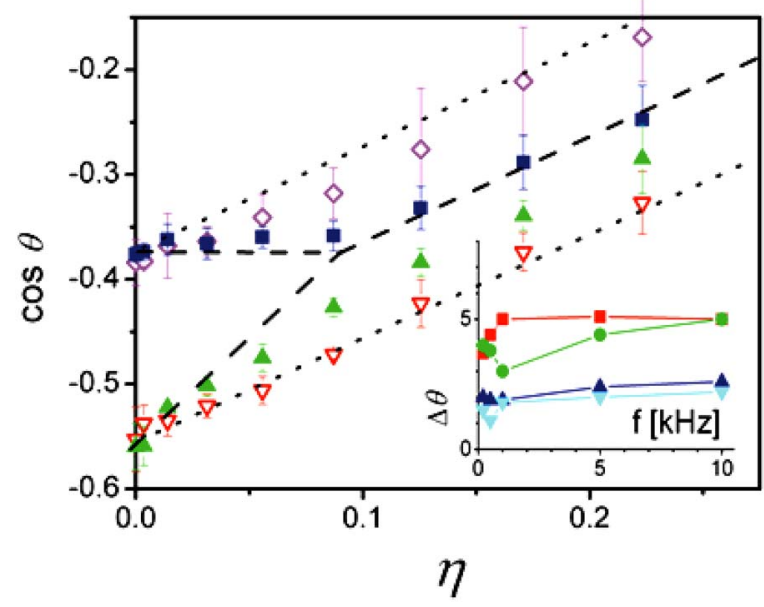

FIG. 3. (Color online) Cosine of advancing and receding c.a.'s vs EW number. Open symbols: dc voltage; filled symbols: ac voltage. Dotted and dashed lines: model predictions for $\mathrm{dc}$ and ac voltages, respectively. Inset: c.a. hysteresis vs ac frequency for fixed values of $\eta=0.01,0.03,0.07,0.12$ (top to bottom).

for $\eta<\tilde{\eta}$ to a smaller but finite value for $\eta \geqslant \widetilde{\eta}$, largely independent of the applied frequency for $\nu \approx 1-10 \mathrm{kHz}$ (see inset of Fig. 3). For dc voltage, the hysteresis is essentially voltage independent. This generic scenario is very robust and was also observed for other substrate materials with higher intrinsic c.a. hysteresis, namely, commercial foils of Teflon and of polyethylene with a maximum hysteresis reduction of $\approx 20^{\circ}$ (data not shown).

To understand these observations, we analyze the balance of forces at the $\mathrm{CL}$ in the usual Young picture. The equilibrium c.a. $\theta_{Y}$ is determined by the balance of horizontal components of the surface tension forces acting on the contact line [see Fig. 1(b)]. In units of $\sigma_{\mathrm{lv}}$, the total horizontal force is given by

$$
f=\frac{1}{\sigma_{\mathrm{lv}}}\left(\sigma_{\mathrm{sv}}-\sigma_{\mathrm{sl}}-\sigma_{\mathrm{lv}} \cos \theta\right)+f_{\mathrm{el}}=\cos \theta_{Y}-\cos \theta+f_{\mathrm{el}}
$$

where $\sigma_{\mathrm{sv}}$ and $\sigma_{\mathrm{sl}}$ are the solid-vapor and the solid-liquid interfacial tension, respectively, and $f_{\mathrm{el}}$ is the total electrostatic force (in units of $\sigma_{\mathrm{lv}}$ ) acting on the CL. For dc voltage, $f_{\mathrm{el}} \equiv \eta$, as can be obtained by averaging the local stresses over a distance of order $d$ around the CL (e.g., Refs. 17 and 18). Equating $f$ in Eq. (2) to zero produces the EW equation, Eq. (1).

On real surfaces, chemical and topographic inhomogeneities give rise to additional pinning forces, with corresponding energy barriers that are typically large compared to thermal energies. To move the CL, $f$ in Eq. (2) has to exceed the maximum pinning forces $f_{p}$. Microscopically, the latter depend on complex distribution of the pinning sites on the surface. Microscopically, however, they manifest themselves in the experimentally observed values of advancing $\left(\theta_{a}^{0}\right)$ and receding $\left(\theta_{r}^{0}\right)$ c.a.'s at zero voltage. Assuming that the pinning forces are not affected by the electric fields, we obtain $\theta_{a}$ and $\theta_{r}$ by equating $f$ to the maximum and to the minimum value of $f_{p}$, i.e., to $\cos \theta_{a}^{0}-\cos \theta_{Y}$ and to $\cos \theta_{r}^{0}-\cos \theta_{Y}$, respectively. Since $f_{\mathrm{el}}=\eta$ for dc voltage, we find that both $\theta_{a}$ and $\theta_{r}$ decrease in the same way with increasing voltage as
icense or copyright; see http://apl.aip.org/about/rights_and_permissions 
the equilibrium contact, i.e., according to Eq. (1). This behavior is represented by the dotted lines in Fig. 3.

The ac case is more delicate since the electrostatic force is time dependent. For a sinusoidal voltage $U(t)=U_{0} \sin \omega t$ $=\sqrt{2} U_{\text {rms }} \sin \omega t$, we have

$$
f_{\mathrm{el}}(t)=\eta(\sin \omega t)^{2}=\eta(1-\cos 2 \omega t),
$$

which contains a dc component equal to $\eta$ and an ac component of amplitude $\eta$ oscillating with $2 \omega$. For frequencies sufficiently far above the lowest eigenfrequency of the drop (several tens of hertz for millimeter sized drops), the drop cannot follow the oscillatory force on a global scale and the apparent c.a. is determined by the time average $\left\langle f_{\mathrm{el}}\right\rangle=\eta$. Contact line pinning, however, takes place on a much smaller local scale, on which the liquid responds much faster than the ac frequencies employed here. Hence, we consider the electrostatic force in Eq. (2) as varying quasi statically between 0 and $2 \eta$. In this case, we assume that the CL is depinned whenever $f$ exceeds the maximum (minimum) pinning force at some moment during the ac oscillation cycle. The voltage-dependent values of $\theta_{r}$ and $\theta_{a}$ can then be determined from Eq. (2) using the minimum $\left(f_{\mathrm{el}}=0\right)$ and maximum $\left(f_{\mathrm{el}}=2 \eta\right)$ of the electric force, respectively. Hence, we obtain the prediction that $\theta_{r}$ is independent of the applied voltage, i.e., $\cos \theta_{r}(U)=\cos \theta_{r}^{0}$, while $\theta_{a}$ decreases such that $\cos \theta_{a}(U)=\cos \theta_{a}^{0}+2 \eta$, in good agreement with the experimental results (dashed lines in Fig. 3). Physically, this asymmetry in the behavior of $\theta_{a}$ and $\theta_{r}$ is quite plausible: $f_{\text {el }}$ pulls along the advancing direction. Hence, it can help the CL to advance, but not to recede.

The model also implies that the c.a. hysteresis decreases linearly with increasing $\eta$ from its initial value $\Delta \cos \theta^{0}$ $=\cos \theta_{r}^{0}-\cos \theta_{a}^{0}$ to zero at a critical value $\widetilde{\eta}=\Delta \cos \theta^{0} / 2$. For $\eta \geqslant \tilde{\eta}, f_{\text {el }}$ is larger than the maximum pinning force and hence c.a. hysteresis is predicted to vanish completely. $\theta_{r}$ and $\theta_{a}$ then decrease along with the equilibrium c.a. following Eq. (1) (see dashed lines in Fig. 3).

The scenario described above obviously captures all the important features of the experimental data. Yet, from a quantitative perspective, some deviations are observed. While the receding c.a. is indeed fairly constant for small $\eta$, the slope of $\cos \theta_{a}$ is less steep than predicted. Furthermore, the experiments still display some residual hysteresis for $\eta$ $\geqslant \widetilde{\eta}$. The field-induced depinning mechanism is thus less efficient than assumed, which we attribute to a combination of several effects. First, the depinning process is certainly not instantaneous. Only if the net force on the contact line ex- ceeds the maximum pinning force over a finite time interval, passage of the CL from one metastable configuration to another is possible. Since the average electrostatic force over a finite time interval is less than $2 \eta$, the slope in Fig. 3 would be reduced accordingly. Second, $f_{\text {el }}$ is known to be distributed over the distance $d .{ }^{16}$ As a consequence, the efficiency of $f_{\text {el }}$ for pinning centers with size much smaller than $d$ will be reduced. Third, an increased "spikiness" of the c.a. data that is sometimes observed at high voltage may indicate that the pinning forces themselves may also change slightly. A detailed analysis of these aspects is beyond the scope of this communication.

In view of the robustness of the phenomenon, we anticipate that the observed reduction of the c.a. hysteresis will find wide applications in facilitating drop and contact line motion in various applications, such as EW-driven lab-on-achip devices and immersion lithography. In particular, we expect that the threshold voltage for drop actuation can be reduced for a suitable ac excitation of the drops.

We thank Dirk van den Ende and Adrian Staicu for intensive discussions. This work was supported by the Institute for Mechanics, Process and Control Twente (Impact) and by the MESA+ institute for nanotechnology at the University of Twente.

${ }^{1}$ E. B. Dussan and R. T. P. Chow, J. Fluid Mech. 137, 1 (1983).

${ }^{2}$ P. G. de Gennes, Rev. Mod. Phys. 57, 827 (1985).

${ }^{3}$ J. F. Joanny and P. G. de Gennes, J. Chem. Phys. 81, 552 (1984).

${ }^{4}$ M. G. Pollack, R. B. Fair, and A. D. Shenderov, Appl. Phys. Lett. 77, 1725 (2000).

${ }^{5}$ S. K. Cho, H. J. Moon, and C. J. Kim, J. Microelectromech. Syst. 12, 70 (2003).

${ }^{6}$ J. Berthier, P. Dubois, P. Clementz, P. Claustry, C. Peponnet, and Y. Foulliet, Sens. Actuators, A 134, 471 (2007).

${ }^{7}$ J. Z. Chen, S. M. Troian, A. A. Darhuber, and S. Wagner, J. Appl. Phys. 97, 014906 (2005).

${ }^{8}$ K. Ichimura, S.-K. Oh, and M. Nakagawa, Science 288, 1624 (2000).

${ }^{9}$ M. K. Chaudhury and G. M. Whitesides, Science 256, 1539 (1992).

${ }^{10}$ R. E. Johnson and R. H. Dettre, J. Phys. Chem. 68, 1744 (1964).

${ }^{11}$ E. L. Decker and S. Garoff, Langmuir 12, 2100 (1996).

${ }^{12}$ C. Andrieu, C. Sykes, and F. Brochard, Langmuir 10, 2077 (1994).

${ }^{13}$ C. Della Volpe, D. Maniglio, M. Morra, and S. Siboni, Colloids Surf., A 206, 47 (2002).

${ }^{14}$ S. Daniel, M. K. Chaudhury, and P. G. de Gennes, Langmuir 42, 4240 (2005).

${ }^{15}$ P. Brunet, J. Eggers, and R. D. Deegan, Phys. Rev. Lett. 99, 144501 (2007).

${ }^{16}$ J. Buehrle, S. Herminghaus, and F. Mugele, Phys. Rev. Lett. 91, 086101 (2003).

${ }^{17}$ F. Mugele and J.-C. Baret, J. Phys.: Condens. Matter 17, R705 (2005).

${ }^{18}$ T. B. Jones, Langmuir 18, 4437 (2002). 